membrane may be divided horizontally behind the growth and the muscular part separated partly by snips of the scissors and partly by tearing until the lingual artery is seen towards the middle line and deeply towards the floor of the mouth. It may generally be defined and caught in forceps before division. In this method of operating the bleeding is easily controlled, and what bleeding there is takes place out of the mouth instead of down the pharynx, and so there is no necessity for a preliminary laryngotomy. If the disease involves the mucous membrane of the mouth anteriorly to a large extent with perhaps adhesion to the jaw it will be well to saw through the jaw in the middle line in front, separate the two halves, remove the disease and wire the fragments together. If the disease is situated far back or involves the floor of the mouth extensively on one side it will be well to attack it from the side of the neck by some such incision as Kocher's, the lingual artery being tied and the glands removed through the same incision. When the lymphatic glands are not removed through the same incision as the tongue they must be attacked at a second operation undertaken two or three weeks after the first. Mr. Butlin strongly advocates the removal of the glands whether they can be felt enlarged or not, as he has found that on opening the deep fascia of the neck glands are often found enlarged and infected that could not be felt at all before the operation. He advises that all four groups of glands previously mentioned should be removed with the connective tissue and the submaxillary salivary gland dissected out in one piece if possible. So far I have dealt only with those cases where there was little doubt that the disease could be removed, but unfortunately we are often confronted with very advanced forms of the disease where it is most difficult to decide whether to advise the patient to submit to operation or not. Death from cancer of the tongue is a most horrible one as will have been gathered from some mentioned symptoms of the final stages already recommed, and the best rule to follow is that be completed by Mr. Butlin. If the disease can be undertaken eradicated in the mouth it should the glands, for death matter what the condition of is far less or death from glandular involvement disease. If the frul than that from the primary be completely extirpated within the mouth cannot for recurrence extirpated it should be left alone condition be will rapidly ensue and the patient's tongue or a portion of improved. After the raw surface left should be been removed, the possible with suitably be diminished as far as parts not sovered arranged sutures, those or paintf covered in being dusted with iodoform balsam in which the spirit has 's varnish, friars' saturated solution seen raced by a after trealution of iodoform in ether. The is not to allow the patient to invalid; let him sit patient to consider himself an from the let him sit up as soon as he is well round out with antiseptic and begin washing his mouth not able to do this. Get him out of bed the day after operation. I believe I have seen old men die after this operation from being kept in bed too long. Plenty of nourishing liquid food and stimulants must be given by means of a tube fastened to the end of a feeder and passed over the back of the tongue or by means of the nasal tube. Should there be any difficulty in this, nutrient enemata may be required during the first twenty-four hours. You will be surprised how well many patients manage to speak, even when the whole tongue has been removed. When the case is inoperable we must do what we can to relieve the patient's sufferings by means of morphia given by mouth or hypodermically, by cocaine applied locally, and sometimes by division, or, better, excision of a portion of the lingual nerve in the mouth. The mouth must be frequently cleansed by washing out or syringing to prevent the swallowing and inhalation of the discharges. Perhaps the $x$-rays may be of use in the relief of pain as they have been in inoperable carcinoma in other regions.

\section{MIXED TUMOURS OF THE SALIVARY GLANDS.}

THE greater number of tumours arising in connection with the salivary glands are of a complex structure, containing as they do a variety of tissues, e.g. cartilage, myxomatous tissue, and fat. Besides these definitely mesoblastic structures they contain cells which may resemble epithelial, endothelial, or connective-tissue cells, and accordingly the tumours have been regarded as of epithelial, endothelial, or sarcomatous nature. Hence it will be perceived that there is a wide divergence of opinion among pathologists with regard to the true nature of these peculiar new growths. Dr. F. C. Wood ${ }^{1}$ has made a careful study of the clinical histories of a large number of cases of tumour formation in connection with the salivary glands, together with details of the microscopic appearances observed in the tumours after their removal. Of the total number (59), 54 he states may be considered as undoubted mixed tumours of the endothelial type. Only four of these were from the lip, four from the pharynx or palate, two from the neck, and one from the cheek. The remainder were from the parotid or submaxillary glands.

\section{General Morphology.}

The mixed tumours of the salivary glands are found, as a rule, to be encapsulated lobular growths, with harder and softer areas, the denser portions being due, as a rule, to the presence of cartilage or firm connective tissue. They can be divided, roughly, into three groups, each having to a certain extent, characteristic morphology and definite clinical course :-(1) Very fibrous tumours with but little cellular structure, and with but little mucous degeneration and no cartilage; (2) Very hard tumours containing large masses of cartilage and but little connective tissue or cellular parenchyma; (3) Soft, very cellular growths with transparent trabeculæ of mucous tissue surrounding areas, which are opaque and yellow, which on 
microscopical examination will be found to be dense cellular areas, the colour being occasionally, though not always, due to fatty degeneration or necrosis of the cells. The first and second forms are likely to be benign in their clinical course, while the third form is apt to recur locally or to run an exceedingly malignant course. A few only of the tumours examined belong to the first class, about one-fourth fall into the second group, while the majority belong to the third. It is important to remember, however, that a great amount of variation is present in different parts of the same tumour, and while one portion may be cellular another may be composed largely of cartilage.

\section{Time of Occurrence.}

The development of these tumours may take place before birth, in which case they are often associated with other congenital defects of the facial region. As a rule, the actual development becomes noticeable during early adult life. The average duration of time between the first discovery of the tumour and operation was eight years and nine months in Dr. Wood's cases. In one the tumour had been present for 53 years when it became malignant.

Situation of Mixed Salivary Tumours.

The growths are seen in the pharynx attached to the bony structures of the hard palate, often indenting the substance of the bone, but not involving the bone substance, from which they are separated by their own fibrous capsule and the periosteum. They also occur on the inner surface of the cheek, usually near the opening of the parotid duct, and in the substance of the lip, most often near the middle line. They are also found in the cervical region in the upper triangle of the neck, in front of the sterno-mastoid muscle. The most frequent situation is near or in the substance of the parotid and submaxillary glands. In the case of large growths the remains of the glandular tissue are stretched out over the tissue of the tumour and undergo pressure atrophy, but are separated from the tumour substance by a capsule. In the pharynx, lips, and cheeks the tumours usually lie just below the surface of the mucous membrane; but the parotid, submaxillary, and cervical new growths are subjacent to the deep fascia. Unless they are large, there is usually considerable mobility. Fixation, if not merely due to the size, is a sign of malignancy. The skin is freely movable over the benign growths.

\section{Clinical Course.}

Out of 37 cases in which the after history is: recorded, 20, or 55 per cent., were permanently cured by operation; 17 , or 45 per cent., recurred locally. In four of these cases fatal recurrence occurred, and in one other there was a secondary growth too extensive for removal. In the remaining 12 there were local recurrences which were satisfactorily dealt with by further operation. Malignancy can be estimated to a certain extent by the rapidity of growth of the tumours and their physical characters, the softer ones being more likely to be malignant; but it not unfrequently happens that a growth which has existed for a long time without showing any signs of malignancy suddenly takes on a rapid growth. infiltrates surrounding tissues, and forms metastases, though it is very seldom that these latter are found in the lymphatic glands.

Dr. Wood concludes his paper with a summary and critical review of the various theories which have been held by pathologists to account for these growths. He himself inclines to the view that they arise in connection with developmental tissue inclusions derived from the branchial arches, and points out in support of this view that where complicated processes of development occur, ihere: especially are complex tumours found, e.q. in kidney, ovary, testicle, and the site of the postanal gut.

\section{Progress in Genito-Urinary Surgery.}

The Functional Diagnosis of Kidney Disease.-F. Engelmann ${ }^{1}$ has been able to prove, as Bickel had previously done, that the electrical conductivity of the urine and blood-serum of healthy and unhealthy individuals is extraordinarily constant. Further, the electrical conductivity of the urine obtained from each kidney by ureteral catheterisation is exactly proportionate to, and varies proportionately with the molecular concentration. For example, if one kidney is diseased, the conductivity is diminished just as the freezing-point is raised (i.e. brought nearer to that of distilled water). Hence, the determination of the electrical conductivity of urine gives excellent control over the results obtained by cryoscopy.

Movable Kidney.-Augustus H. Goelet (New York) ${ }^{2}$ describes a technique for fixation of the prolapsed kidney which he has employed in 171 consecutive nephropexies on 134 patients, in 37 of the cases for both kidneys at the same time. In this series of cases there was no death and no failure to secure sufficient fixation of the kidneys.
The following points are considered by the author to be essential for success in nephropexy by any method, namely :- (1) Careful preparation of the patient beforehand to prevent retching after the operation. (2) Particular care in completely detaching the colon from the kidney, to prevent subsequent dragging upon it when the bowel becomes distended. (3) Prevention of re-attachment of the colon to the kidney which may be secured by packing a gauze drain below and in front of the kidney, and by evacuating the bowels early. (4) The sustaining sutures must be inserted under the fibrous capsule in such a manner as to prevent them tearing out or cutting through when tied, and the suture material must not stretch or absorb before. the kidney becomes fixed. (5) The kidney must be restored by the operation to its normal position, not to a lower position than normal. (6) The fatty capsule must be prevented from coming between the kidney and the structures to which it is sutured. This the author prevents by packing. gauze around the lower pole of the kidney. The 